\title{
Influence of a wet wood particle form on the characteristics of its ignition in the high-temperature medium
}

\author{
G.V. Kuznetsov, S.V. Syrodoy*, N.Y. Gutareva \\ National Research Tomsk Polytechnic University, 30 Lenin Avenue, Tomsk 634050, 6340050, Tomsk, Russia
}

\section{A R T I C L E I N F O}

\section{Article history:}

Received 19 January 2018

Received in revised form

24 February 2019

Accepted 9 April 2019

Available online 7 June 2019

\section{Keywords:}

A particle of wet wood

Heat and mass transfer

Ignition of volatiles

Ignition

A particle form

\begin{abstract}
A B S T R A C T
The results of the experimental and theoretical studies of the processes of the single particles ignition of the wood biomass of various forms (a cube, a cylinder and a sphere) have been presented. According to the results of the experimental studies, it has been established that the particles form can have a fairly significant effect on the characteristics and conditions of ignition. It has been shown that the wood particles in the form of a sphere are ignited much faster than cylindrical and cubic ones (ignition delay times are less by 56\%). Such a tendency is maintained for particles sizes from $1 \cdot 10^{-3} \mathrm{~m}$ to $3 \cdot 10^{-3} \mathrm{~m}$. According to the results of the experiments, the mathematical model for ignition of a particle of wet wood biomass has been formulated, taking into account the joint occurrence of the main processes of the thermal preparation (radiation-convective particle heating, evaporation of water, thermal decomposition of the organic part of the fuel, output, ignition and combustion of gaseous pyrolysis products, ignition of the char residue).

The formulated system of the nonlinear differential equations in the partial derivatives with the corresponding boundary conditions describes the ignition process in a two-dimensional setting, taking into account the substantial nonuniformity of the temperature field of a wood particle in the induction period. We have taken into account that the evaporation process takes place in the front. Up to the present time, the mathematical models of such a degree of detailing of the physical processes occurring together when igniting wet wood have not been formulated. A comparative analysis of the ignition delay times $\left(t_{i g n}\right)$ obtained theoretically and experimentally has showed their satisfactory correspondence. As a result of the numerical simulation, the temperature fields in the system "a fuel particle - hightemperature medium" have been obtained, illustrating the high gradients of the temperatures at the moment of ignition. It has been established that it is possible to ignite the water-saturated wood particles before they dry completely. It has been established that the ignition mode of wood particles is possible before they dry completely.
\end{abstract}

(C) 2019 Elsevier Ltd. All rights reserved.

\section{Introduction}

\subsection{Economic feasibility of the wood biofuel use}

The analysis of the world tendencies [1-3] in the development of the fuel and energy complex shows that the wood biomass is becoming increasingly important as a basic fuel for large (thermal power plants) and small (local boiler stations) energy. Such a trend is typical for countries of Western Europe (Germany, Denmark,

\footnotetext{
* Corresponding author. Lenin Avenue, 30, housing 4, audience 203, Tomsk, 634050, Russia.
}

E-mail address: ssyrodoy@yandex.ru (S.V. Syrodoy).
Sweden) [4-6], North (the USA and Canada) [7] and South America (Brazil) [8], and South Asia (India) [9]. For example, the most modern thermal power plant Avedore (Denmark) [10] from the second half of 2016 was transferred completely to burning biomass. The popularity of wood and other types of biomass is explained, first of all, by the presence of a number of undoubted advantages in comparison with the traditional solid (coal), liquid (fuel oil) and gaseous (natural gas) types of energy carriers [12-15]. First of all, wood is the only renewable type of fuel [12].

The undoubted advantage of using wood is that due to the relatively low combustion temperature (up to $1200 \mathrm{~K}$ [13]), when it is burned, much less (approximately 5 times) of oxides of nitrogen and sulfur are formed than in the oxidation of coal [14]. It should also be noted that woodworking industry waste can be used as raw 
material for fuel production based on wood biomass [15], the volumes of which are large.

After having analyzed literature [1-15], it can be concluded that the most appropriate is the use of wood as a solid fuel for smallsized boiler units, and in the medium term and large thermal power plants. Most likely, direct burning of wood biofuel in the furnace devices of the boiler units is the most promising (at the present time) and the most efficient method of using wood biomass in power engineering [16]. At the same time, until now no mathematical models have been developed that describe the complex of the thermal preparation processes of wet, as a rule (to a greater or lesser extent) wood to ignition, occurring together in the induction period of time. This is due to the fact that the ignition of wood particles occurs under conditions of the intense phase and thermochemical transformations.

It is known [17] that wood waste generally has a high humidity (up to $60 \%$ by weight). Accordingly, it can reasonably be assumed that one of the most significant stages in the thermal preparation of fuel for combustion is its drying. But for carrying out the procedure of fuel dehydration it is necessary to use special drying equipment. Fuel dryers, as a rule, are energy-intensive equipment occupying a vast space inside the thermoelectric power station (TPS). Also, when drying the wood, it is necessary to use a drying agent and pipelines for its delivery. For this reason, the most appropriate is the wet wood burning in the furnace space of the boiler unit by arranging in it an aerodynamic regime with a constant recirculation of the torch by analogy with [18].

It can be noted that reducing the influence of the internal moisture of the wood on the dynamics of ignition due to the increase in the degree of grinding (reduction in the size of the fuel particles) will lead to a significant increase in the energy consumption for grinding fuel. For this reason (as it is stated in Ref. [19]), the most optimal size of biomass particles is $2-3 \mathrm{~mm}$. At such sizes of the fuel particles, the influence of the evaporation processes inside the pore moisture and the temperature distribution along the particle radius on the ignition dynamics becomes significant. It can also be said that for typical sizes of fuel particles $\delta \sim 3 \cdot 10^{-3} \mathrm{~m}$, a significant factor affecting the rate of ignition may be a particle form. This is due to the peculiarities of the formation of the temperature fields in the objects of different configurations [20]. When grinding solid fuel (particularly wood), as a rule, irregularly formed particles with an arbitrary number of faces are formed. For this reason, for assessing the conditions for the ignition of wood, it is advisable to investigate particles of three typical forms - a cylinder, a sphere and a cube.

\subsection{A brief review of the experimental studies of the wood particles ignition}

The presence of a group of factors (dispersity, type of wood, humidity, a particle form, etc.) that affect the dynamics of the single wood particles ignition makes the systemic experimental studies of the ignition processes expensive. For this reason, in spite of the conducted researches [21-24] of the processes of wood ignition, the general theory of thermal preparation, ignition and burning of wood-fuel particles has not been developed to date. For example, in Ref. [21], the results of the experimental studies of ignition biomass particles of two characteristic sizes (80-90 $\mu \mathrm{m}$ and $224-250 \mu \mathrm{m}$ ) have been presented. The experiments have been performed on a quartz hollow cylinder installation mounted on a McKenna flat flame burner. The particles of wood have been delivered into the cavity of the cylinder. The entire process has been recorded by a high-speed camera. As it has already been mentioned above, the grinding of wood has been carried out to a size of $0.08-0.25 \mathrm{~mm}$. But obtaining wood dust such a degree of grinding is a very energyconsuming process. As it has been established in Ref. [19], the optimal particle size for burning is $2-3 \mathrm{~mm}$.

When studying the particles of such small dimensions $(0.08-0.25 \mathrm{~mm})$, it is impossible to estimate the effect of the form on the characteristics of the ignition.

The results of the experimental studies of the ignition of the wood particles of two groups have been given: large - up to $3 \mathrm{~mm}$, and small $\sim 210 \mu \mathrm{m}$ in Ref. [22]. The large particles have been cubes $(3 \times 3 \times 3 \mathrm{~mm})$ based on an olive tree or pine, or Miscanthus needles $(3 \times 3 \mathrm{~mm})$. The particles have been placed in a cuvette (basket) located inside the water-cooled housing. Such a design allows minimizing "extraneous" (in other words, reducing the effect of thermal noise or interference) thermal effects (radiation from the walls of a high-temperature furnace) on the wood particles when conducting the experimental studies. The temperature of the wood has been measured with a thermocouple.

According to the results of the experimental and theoretical studies it has been found that the particle size also has a significant effect on the rate of ignition. Thus, the analysis of Figs. 7 and 8 shows that reducing the size of the cubic particles from 3 to $1 \mathrm{~mm}$ (in three times) leads to a decrease in the ignition delay time by 2.3 times (from $\approx 22$ to $\approx 9.5 \mathrm{~s}$ ). But it is worth noting that a comparative analysis of the ignition of the wet wood particles (with a characteristic size of $3 \mathrm{~mm}$ ) of cubic, cylindrical and spherical forms (provided that the volume of particles is identical) has shown the tign values decrease from $\approx 22$ to $\approx 10$ s (or words, in 2.2 times). Accordingly, it can be concluded that the form of the wood particle has a comparable (in terms of importance) effect on the characteristics and conditions of ignition.

As a result, the ignition delay times have been determined. The ambient temperature ( $\mathrm{Tg}$ ) has been varied [22] in the range from 490 to $670 \mathrm{~K}$. In this case, the main task of the study [22] has been to determine the minimum $\mathrm{Tg}$ value sufficient for igniting wood. The effect of the particle form on the ignition characteristics has not been studied. However, the conditions of heat transfer in the cuvette (in the layer of chopped wood) differ significantly from similar conditions when spraying biomass particles in the combustion space. Therefore, the use of the results of [22] in the design of the boiler equipment for burning wood has not been sufficiently justified.

A large amount of the experimental data on the ignition of pellets of a spherical form is given in Ref. [23]. However, the test fuel was a mixture of finely dispersed wood and coal. Obviously, the process of ignition of such a fuel composite will be significantly different from the ignition of woody biomass particles. Also in Ref. [23], the influence of the particle form on the dynamics of ignition has not been investigated.

In Refs. [21-23], the processes of the dry wood particles ignition have been investigated. But it is known [17] that the real biomass always contains moisture, which can have a significant effect on the characteristics and conditions of ignition [25]. It should also be noted that in Refs. [21-23] the experiments have been carried out only in a small range of ambient temperatures (from 800 to $900 \mathrm{~K}$ ). Such low temperatures are not typical for steam and hot water boilers. But, the studies [21-23], despite the limited potential of the application of the results, allow us to make several useful conclusions for the further study of the laws of the wood particles ignition. Thus, for example, the high thermal inertia of the process of the even dry wood ignition is evident, which gives grounds for the hypothesis that the form of the particle must significantly influence the characteristics and conditions of its ignition. 


\subsection{A brief review of the mathematical models of the wood particles ignition}

To date, several (most important) works have been published with the results of the theoretical studies of the processes of wood ignition [25-27]. But most of the previously formulated mathematical models describe the ignition, as a rule, of dry particles. Such a representation allows not to take into account the evaporation of the intraporous moisture and the change in the thermophysical characteristics of the wood in the process of its dehydration.

The mathematical models of ignition of single particles of wood fuel developed to date [25-27], as a rule, are based on the solution of the equations of mathematical physics in a one-dimensional formulation. For example, in Ref. [25] the results of the numerical modeling of the ignition of the wood layer are presented, which have been obtained by solving a one-dimensional heat equation. In this case, the evaporation of moisture (both intraporous and bound) in Ref. [25] has not been considered. The use of the onedimensional statement of the ignition problem by analogy with [25] for analyzing the effect of the geometry of a fuel particle on ignition characteristics is impossible.

In Ref. [26], the results of the experimental and theoretical investigations of the biomass layer ignition with allowance for the presence of intraporous moisture have been presented. At the same time, a complicated complex of the thermochemical (pyrolysis of solid fuel, gas-phase ignition of thermal decomposition products) and thermophysical (evaporation of moisture, radiative heat transfer) processes have been taken into account. But in Ref. [26], the ignitability of the biomass layer has been investigated under conditions corresponding to a treetop forest fire. Another distinguishing feature of [26] is that the evaporation of moisture has been considered in the context of ideas about the dehydration process as a chemical reaction. Under such conditions, the intensity of moisture evaporation is described by a simple Arrhenius dependence. But in fact the evaporation of moisture flows in a very narrow zone, constantly moving along the particle (the front of evaporation) [27]. At the same time, the velocity of this front depends greatly on the temperature of its surface.

In mathematical models [25,26], the effect of the fuel particle form on the dynamics of ignition has not practically been taken into account. There are known works [28-30] devoted to the analysis of the influence of wood pellets on the characteristics of pyrolysis. So in Ref. [28] the results of mathematical modeling of the thermal decomposition process of wood particles of the cylindrical form have been resulted. At the same time, the process of pyrolysis of fuel has been considered with taking into account the staged nature of the thermal decomposition process. The processes of filtration of pyrolysis products through porous wood structure have also been taken into account. But in Ref. [28], the ignition processes of the gaseous products of pyrolysis in the gas phase have not been simulated, and the analysis of the effect of the particle form on the characteristics and conditions of the wood ignition has not been carried out.

Another example is the model [29], in which the complex of thermochemical (thermal decomposition of wood with the formation of volatile and char) and the thermophysical (radiationconvective heating, evaporation of water) processes have been taken into account that occur together in the pyrolysis of wood particles. At the same time, the influence of three forms (a sphere, a cylinder and a cube) on the dynamics of thermal decomposition has been analyzed. But the ignition processes have not been considered in Ref. [29], and the ignition delay times, respectively, have not been determined, although a rather complicated complex of the thermophysical processes taking place together has been considered in Ref. [29]. The mathematical model [29] is based on the solution of the balanced (zero-dimensional) energy equation. The change in temperature inside the particle has not been taken into account. The application of such an approximation to the particle of biomass under conditions of high-temperature action (for example, upon ignition in the conditions of a combustion chamber) is not justified. It is also worth noting that the evaporation model given in Ref. [29] is based on the assumption that the temperature is at equilibrium at the boundary of the evaporation front. As it has already been mentioned earlier, such a representation is physically incorrect. Water evaporates at any temperature above the triple state of the substance $(273.16 \mathrm{~K})$. It is also worth noting that in the model [29] the heat runoff has not been taken into account resulting from the evaporation of water.

The most complete mathematical model of the pyrolysis process has been in Ref. [30]. The process of pyrolysis of an ellipsoidal particle of wood biomass has been considered. It has been assumed that the process of thermal decomposition proceeds with the formation of solid, liquid and gaseous products of thermal decomposition. The filtration of the thermolysis products through the porous structure of the particle has also been taken into account. But in Ref. [30] thermal decomposition of dry wood particles has been investigated. The evaporation of moisture and the influence of the form factor on the dynamics of pyrolysis have not been taken into account.

Summing up, it can be said that despite the extensive studies of the processes of pyrolysis and ignition of wood particles [21-30], no experimental or theoretical studies of the influence of the form of woody biomass particles on the characteristics and conditions of ignition have so far been carried out. Therefore, the purpose of the work is an experimental and theoretical investigation of the particles ignition of moist wood biomass of various forms on the example of the most typical geometric bodies (a sphere, a cube and a cylinder).

\subsection{Justification of the prospects of research theme}

From the theory of ignition and combustion of the solid fuels [31,32] (including wood [33]), it is known that the dynamics of the ignition processes are determined by the influence of many factors (fuel particles size, moisture content and type of wood, particles form). At the same time, according to B.D. Spoding [31] and D.A. Frank-Kametsky [33] the dynamics of its thermal decomposition has the strongest influence on the process of ignition of the solid fuel and, accordingly, the output of volatiles. At the same time, as it has been established by the results of the experimental [34,35] and theoretical $[28,29]$ studies, the most intensive process of thermal decomposition (respectively, and the yield of the gaseous pyrolysis products) occurs in tree particles in the form of a sphere, then in a cylinder form and cubes. In this case, we can say that the characteristics and conditions of the process of ignition of the wood particles, which always takes place in the gas phase, will directly depend on a form of a particle.

According to the results of the experimental and theoretical studies, it has been established that the particle size also has a significant effect on the rate of ignition. So the analysis of Figs. 7 and 8 shows that reducing the sizes of cubic particles from 3 to $1 \mathrm{~mm}$ (in three times) leads to a decrease in the ignition delay time by 2.3 times (from $\approx 22$ to $\approx 9.5 \mathrm{~s}$ ). But it is worth noting that a comparative analysis of the ignition of the wet wood particles (with a characteristic size of $3 \mathrm{~mm}$ ) of cubic, cylindrical and spherical forms (provided the identity of the volumes of the particles) has shown that the $t_{\text {ign }}$ values decrease from $\approx 22$ to $\approx 10 \mathrm{~s}$ (or in other words, in 2.2 times). Accordingly, it can be concluded that the form of the 
wood particle has a comparable (in terms of importance) effect on the characteristics and conditions of ignition.

As the results of the research [36] have shown, the humidity of the fuel also has a significant impact on the characteristics and conditions of ignition. So, at the external temperature $T_{g}=1073 \mathrm{~K}$, the ignition delay time of a particle (with a characteristic size $\mathrm{d}=3 \mathrm{~mm}$ ) of moist wood decreases from $12 \mathrm{~s}$ to $0.5 \mathrm{~s}$ with the decrease in humidity $(\varphi)$ from $60 \%$ to $0 \%$ (in other words, in 24 times). But burning the wet wood particles is technologically and economically more beneficial than oxidizing pre-dried $(\varphi=0 \%)$ due to exclusion from the technological cycle of preliminary thermal preparation of fuel (drying) of the large-sized equipment (the drying units) and metal structures of the transportation systems of the drying agent (high-temperature gas-air ducts). For this reason, it can be said that the particle form factor is comparable in its significance with the wood moisture factors and the size of the fuel particles.

\section{Experiment}

The experimental studies have been carried out on the stand, the scheme of which is shown in Fig. 1. A particle of wood has been fixed on a metal holder and introduced by a remotecontrolled coordinate device into a hollow ceramic cylinder whose inner surface has been to a high temperature. The registration of the processes of heat and mass transfer and ignition of the fuel particle has been carried out by a high-speed Photron FASTCAM CA4 5 video camera (video-recording speed up to 20,000 frame/s). The air temperature inside the cylinder $\left(T_{g}\right)$ in the experiments has varied over a fairly wide range (from $870 \mathrm{~K}$ to $1270 \mathrm{~K}$ ). The time period from the onset of the thermal action (the moment when the particle has been introduced into the cylinder cavity) prior to ignition (the appearance of a flame) has been considered to be the ignition delay time $\left(t_{\text {ign }}\right)$. The ignition of the particles of two sizes has been investigated: $\delta=1 \cdot 10^{-3}$ and $\delta=3 \cdot 10^{-3}$. To determine the timing characteristics of the ignition process, a series of the experiments has been performed from 10 experiments for each $T_{g}$ value. The processing of the experimental results has been carried out using the method of smallest quadrates [37]. The number of the experiments could vary (but not less than 10) in order to achieve the required accuracy of the determination of $t_{i g n}$. The systematic error in determining the main measured parameters $\left(T_{g}, t_{i g n}\right)$ has been no more than $5 \%$. The confidence interval for determining $t_{\text {ign }}$ at a confidence probability of 0.95 has not been exceeded $17 \%$.

\subsection{Experimental samples}

The studies have been carried out for the particles made from Siberian larch. The structure of this wood provides, in contrast, for example, from birch or spruce, a stable configuration of the particle when it is processed before the experiment. The thermophysical characteristics are given in Table 1 . In the experiments, the particles cut from a monolithic dry fragment of a large-sized wood have been used. Later, with the aid of a grinding device, the surface of the particles has been prepared to give them an appropriate form (a cylinder or a cube). The particles have been then weighed to determine the dry mass. At the next stage of the preparation of the experiment, they have fallen into the water and kept there for several hours. After the extracting period (about $10 \mathrm{~h}$ ), we have reweighed to determine the moisture content.

It is known [17] that the moisture content of freshly cut wood (in particular, larch) is $\varphi$ from 30 to $60 \%$. In the experiments, the particles with a moisture content of $\varphi \approx 40 \%$ have been used. Such a value of $\varphi$ is, most likely, the highest possible in real fuel preparation conditions.

\section{Results of the experiment}

In Fig. 2 the frames of a typical video of the wood particle ignition process (with a characteristic size $\delta=3 \cdot 10^{-3} \mathrm{~m}$ ) of a cubic form are given. The ambient temperature is $T_{g}=1270 \mathrm{~K}$. The total time from the start of heating to the instant of ignition has been $t_{\text {ign }}$ $\approx 2.59 \mathrm{~s}$ in this experiment. The analysis of the frames shows that the process of thermal preparation of wood can be divided conditionally into a series of the successive stages.

After introducing the particle into the high-temperature medium, the intensive heating of the fuel (frame a) and evaporation of water begin. One can see clearly an increase in the brightness of the particle surface. Heating of the fuel leads to thermal decomposition of the material (frame b). A detailed (at a large magnification) analysis of frames of type $b$ shows a characteristic darkening of the particle surface. This is due to the intensive pyrolysis of wood with the formation of gaseous and solid products. It can be noted that the formation of the coke residue in the direction across the fibers forming the wood flows more intensively than along them. The latter is most likely due to the fact that the thermal conductivity of the wood along the fibers is much greater than across them.

The ignition occurs (frame $c$ ) of the volatiles at $t=t_{\text {ign }}$, after the start of the particle heating. After the ignition of the volatiles, the sphere of flame is formed (frame d), whose size is $2-3$ times higher

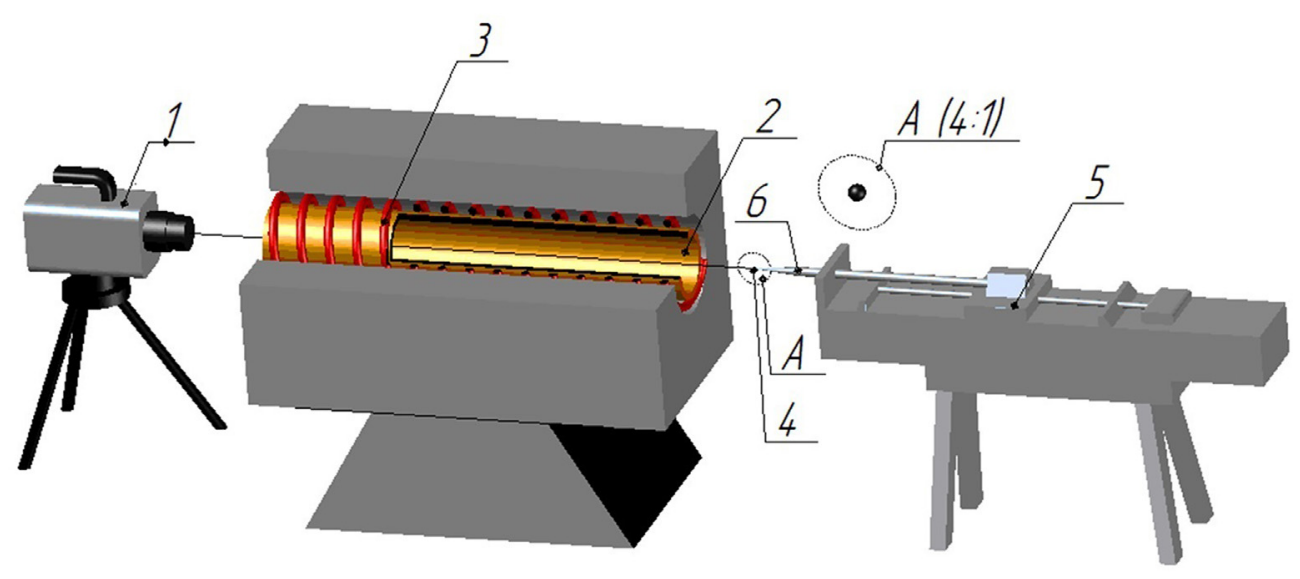

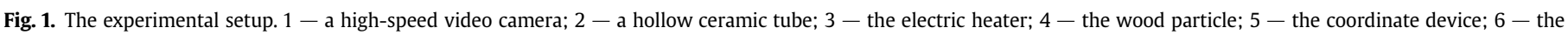
metal holder. 


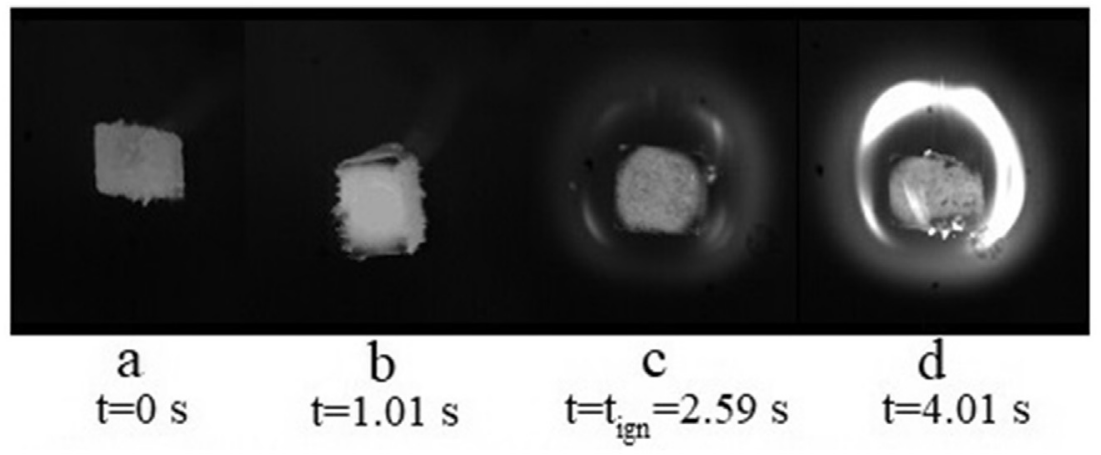

Fig. 2. Typical frames of the video of the process of ignition of particles of moist wood cubic form.

than the initial particle size. Based on the results of the experiments, the delay times for the ignition of the wet wood particles (from 2 to $25 \mathrm{~s}$ ) have been determined, depending on the sizes and form.

Fig. 3 shows the typical frames of the wet wood particles ignition in the form of a cylinder. The analysis of the video frames shows that, like the cube (Fig. 2), it is possible to distinguish the characteristic stages of the ignition process (inert heating, evaporation of moisture, thermal decomposition and ignition).

According to the results of the analysis of the videograms, the physical and mathematical models describing the complex of the processes taking place together (heat transfer by heat conduction, evaporation of water, thermal decomposition of fuel, interaction of water vapor with carbon of char, gas-phase ignition of pyrolysis products), thermal preparation and ignition of wood particles of three forms (a sphere, a cylinder and a cube) and sizes (from $1.5 \cdot 10^{-3}$ to $\left.3.0 \cdot 10^{-3} \mathrm{~m}\right)$.

\section{Statement of the problem}

\subsection{Physical statement}

The particle of wet wood is introduced into a high-temperature environment (Fig. 1.) at the initial moment of time. As a result of the intensive radiation-convective heating, the process of water evaporation is initiated. The evaporation front moves from the surface layers of the fuel to the deep. As a result, a substantially heterogeneous inhomogeneous structure is formed, consisting of wet and dry wood.

Heating of dry wood leads to its thermal decomposition with the formation of gaseous (volatile) and solid (coke) pyrolysis products. The latter leads to an increase in the porosity of the fuel. Water vapor formed during evaporation is filtered through a layer of dry wood, entering into thermochemical interaction with coke carbon. As a result, combustible reaction products $\left(\mathrm{CO}\right.$ and $\left.\mathrm{H}_{2}\right)$ are formed. The latter, together with volatile and water vapor, are filtered to the surface of the fuel. A vapor-gas mixture is formed in a small neighborhood of the particle, the combustion of which begins at the critical temperatures and concentrations. The period of time from the beginning of heating to intense heat release as a result of the chemical reactions of combustion and an exponential increase in temperature is considered to be the ignition delay time. In this case, the heat released by the chemical reactions of oxidation of pyrolysis products exceeds the heat coming from the heating source (external environment).

\subsection{Mathematical formulation of the problem}

The mathematical formulation (the system of the partial differential equations with the corresponding boundary conditions) of the problem of igniting wood according to the basic equations (and solution methods) corresponds to the statements [38-40] (when describing the ignition of the spherical particles of wood) and [41] (when describing the ignition process wood in the form of a cube). The main difference between the statement of the ignition problem, the solution of which is given below, from the models [38-41] is to conduct a comparative analysis (under identical heating conditions) of the temporal characteristics of the processes of ignition of the wet wood biomass particles of three typical forms: a cube, a cylinder, a sphere.

The area of solving the problem of igniting of the wood particle for an example of a spherical form is shown in Fig. 4. The particle of wood during the induction period can be divided into two zones. The first one is the original fuel that is still saturated with moisture,

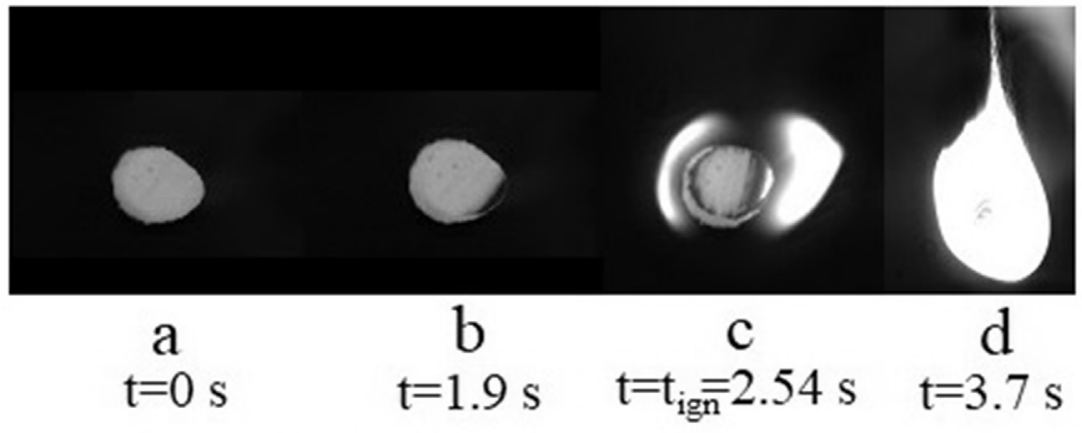

Fig. 3. Typical frames of the video of the ignition process of wet wood particles of cylindrical shape. 


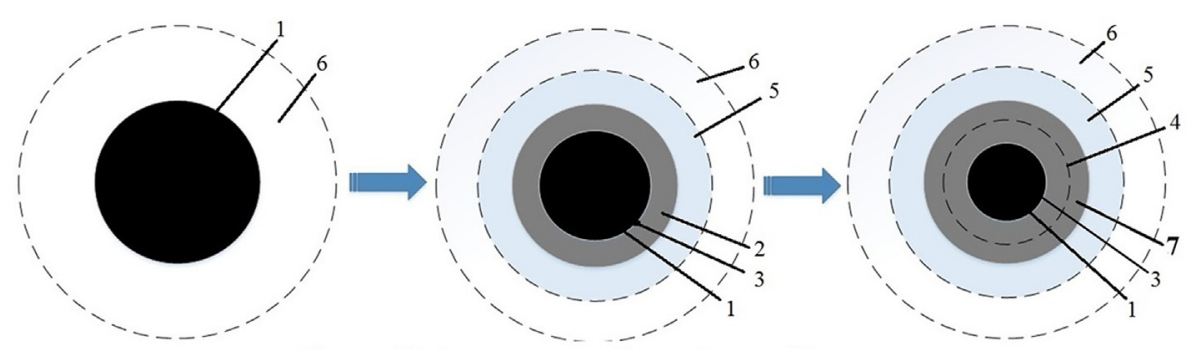

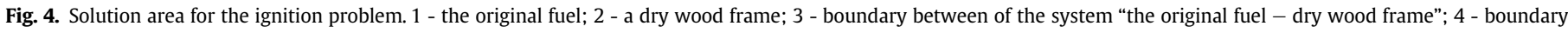

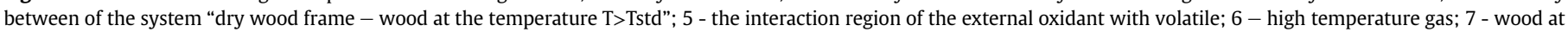
the temperature $\mathrm{T}>\mathrm{Tstd}$.

the second (Fig. 4) is dry wood. The temperature distribution in such a heterogeneous inhomogeneous system is described by the energy equation for a particle, which takes into account water evaporation, thermal decomposition of fuel, thermochemical interaction of water vapor and carbon, as well as the movement of water vapor and thermal decomposition products through a porous framework:

$$
\begin{aligned}
& \frac{\partial H_{w}}{\partial t}+C_{s} \rho_{s} U_{s} \nabla(T) \Phi\left(\xi_{\text {eva }}\right)=\operatorname{div}(\lambda \nabla T)-\frac{Q_{e v a} \rho_{w a t}}{h_{s p r}} \frac{d \xi_{\text {eva }}}{d t} \delta\left(\xi_{\text {eva }}\right) \\
& \quad-\sum_{i=1}^{i=4} Q_{i} \cdot W_{i} \\
& \sum_{i=1}^{n=4} Q_{i} \cdot W_{i}=Q_{w \rightarrow \text { char }} \cdot W_{w \rightarrow \text { char }}+Q_{w \rightarrow v o l} \cdot W_{w \rightarrow \text { vol }} \\
& \quad+Q_{w \rightarrow \text { tar }} \cdot W_{w \rightarrow \text { tar }}+Q_{C+\mathrm{H}_{2} \mathrm{O}} \cdot W_{C+\mathrm{H}_{2} \mathrm{O}},
\end{aligned}
$$

The thermophysical characteristics $\left(\lambda_{w}, C_{w}\right.$ and $\left.\rho_{w}\right)$ have been calculated taking into account the position of the evaporation front. The temperature distribution in the gas layer around the particle is described by the energy equation for a thin layer of the environment around the particle. The latter takes into account the exothermic effects of the oxidation reactions of the main gaseous combustible components with air:

$$
\begin{aligned}
& \frac{\partial H_{g}}{\partial t}+U \nabla H_{g}=\operatorname{div}\left(\lambda_{g} \nabla T\right)+\sum_{i=1}^{i=3} Q_{i} \cdot W_{i} \\
& \sum_{i=1}^{n=3} Q_{i} \cdot W_{i}=Q_{\mathrm{CH}_{4}+\mathrm{O}_{2}} \cdot W_{\mathrm{CH}_{4}+\mathrm{O}_{2}}+Q_{\mathrm{CO}+\mathrm{O}_{2}} \cdot W_{\mathrm{CO}+\mathrm{O}_{2}}+Q_{\mathrm{H}_{2}+\mathrm{O}_{2}} \cdot W_{\mathrm{H}_{2}+\mathrm{O}_{2}}
\end{aligned}
$$

Water vapor together with the gaseous products of thermal decomposition of wood move through the porous wood frame. The pressure distribution over the particle radius has been calculated by solving the piezoconductivity equation [30]:

$$
\begin{aligned}
& \frac{\partial \Pi p}{\partial t}=\operatorname{div}(\chi \nabla P)+\frac{1}{\rho \beta} \sum_{i=1}^{n=3} f_{i} \\
& \sum_{i=1}^{n=3} f_{i}=\frac{1}{s}\left[s W_{t d}+W_{C+H_{2} O \rightarrow C O+H_{2}}+W_{\text {eva }} \delta\left(\xi_{\text {eva }}\right)\right]
\end{aligned}
$$

$f_{i}$ is the function describing the change in mass flow rate of the mixture of water vapor and pyrolysis gases as a result of the release of volatile and absorption of water vapor during its thermochemical interaction with carbon.
Thermal decomposition of the fuel with the release of volatile begins when the condition $T_{\text {sur }} \geq T_{\text {std }}$ reaches the surface of the particle ( $T_{\text {sur }}$ is the surface temperature of the particle, $T_{\text {std }}$ is the temperature of the onset of thermal decomposition (according to Ref. [42])). Thermal decomposition is a complex physico-chemical process that can proceed in several stages (up to 5) [43] with the formation of liquid (tar), solid (coke) and gaseous (volatile) products. As it has been shown in Ref. [43], under the condition of rapid heating (which corresponds to heating in the conditions of the combustion chambers of the boiler units), the process of thermal decomposition of wood is described quite well in the framework of the one-stage model (Fig. 5), with the formation of three pyrolysis products.

The process of thermal decomposition in this case is described by the chemical kinetics equation (by analogy with [43]):

$\frac{\partial \eta_{i}}{\partial \tau}=\left(1-\eta_{i}\right) \cdot k_{i} \cdot \exp \left(-\frac{E_{i}}{R \cdot T}\right)$

In the formulation of the problems, the process of the influx of the products of thermal decomposition and chemical interaction of the solid products of pyrolysis of wood and water vapor into the boundary layer of the particle has been taken into account. As a result, a gas mixture is formed that is flammable when the critical temperatures and fuel concentrations are reached [44]. The time period from the beginning of the thermal effect on the particle to

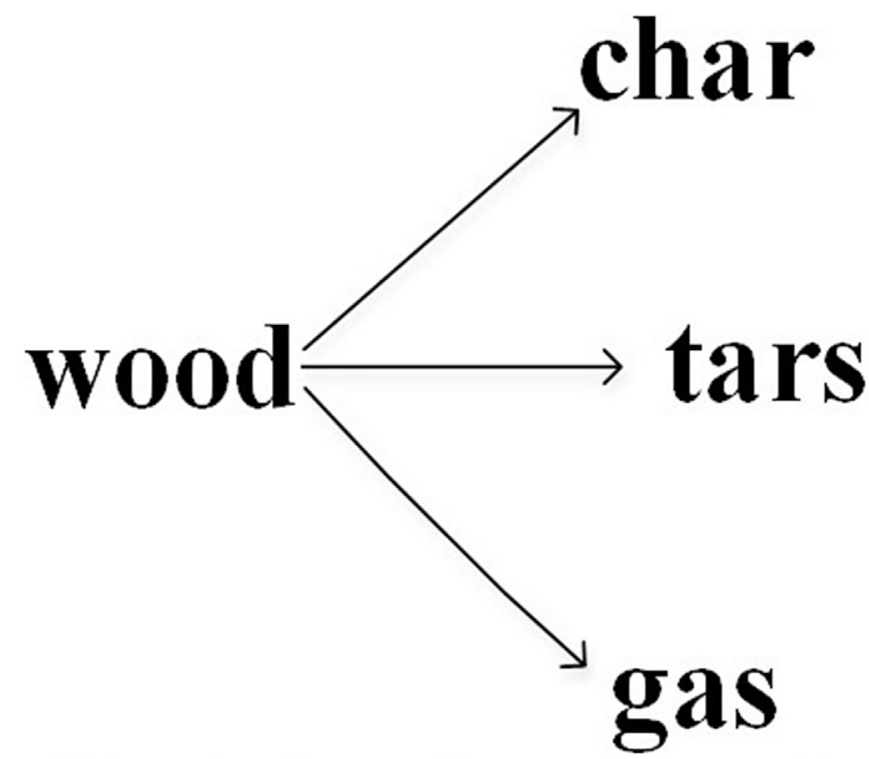

Fig. 5. The mechanism of wood pyrolysis. 
Table 1

Thermophysical properties of wood [46].

\begin{tabular}{|c|c|c|c|}
\hline Temperature $(\mathrm{K})$ & $\begin{array}{l}\text { Coefficient of thermal conductivity }(\lambda) \\
\qquad W /(m \cdot K)\end{array}$ & $\begin{array}{l}\text { Heat capacity (C) } \\
\mathrm{J} /(\mathrm{kg} \cdot \mathrm{K})\end{array}$ & $\begin{array}{l}\text { density }(\rho) \\
\mathrm{kg} / \mathrm{m}^{3}\end{array}$ \\
\hline 373 & 0.18 & 1246 & 499.56 \\
\hline
\end{tabular}

the beginning of the intensive chemical interaction of the vapor-gas mixture with the oxidizer is considered to be the ignition delay time. During ignition, the amount of heat released as a result of oxidation of the fuel must exceed the heat coming from the heat source (the external environment). In the immediate vicinity from the particle surface, the course of the three most significant reactions has been taken into account:

1. $\mathrm{H}_{2}+0,5 \mathrm{O}_{2}=\mathrm{H}_{2} \mathrm{O}+224 \mathrm{~kJ} / \mathrm{mol}$

2. $\mathrm{CO}+0,5 \mathrm{O}_{2}=\mathrm{CO}_{2}+286 \mathrm{~kJ} / \mathrm{mol}$

3. $\mathrm{CH}_{4}+2 \mathrm{O}_{2}=2 \mathrm{H}_{2} \mathrm{O}+\mathrm{CO}_{2}+803 \mathrm{~kJ} / \mathrm{mol}$

The concentrations of the vapor-gas mixture components $\left(\mathrm{H}_{2}\right.$, $\mathrm{CO}, \mathrm{CH}_{4}, \mathrm{CO}_{2}$ and $\mathrm{H}_{2} \mathrm{O}$ ) in the boundary layer of the particle have been calculated by solving the diffusion equations:

$\rho_{g} \frac{\partial c_{i}}{\partial t}+\rho_{g} U \nabla c_{i}=\operatorname{div}\left(D_{i} \nabla c_{i}\right) \pm \sum_{i=1}^{i=3} W_{i}$

As it is known [45], during the thermal decomposition of solid fuel, there is a change in density $(\rho)$ and porosity $(\Pi)$ of the main layer of fuel. Under these conditions, when formulating the problem, the change in $\rho$ and $\Pi$ of the fuel has been taken into account:

$\frac{\partial \rho}{\partial t}=\left(\rho-\rho_{e t d}\right) \cdot k_{e t d} \cdot \exp \left(-\frac{E_{e t d}}{R \cdot T}\right)$,

$\Pi_{p}=\Pi_{0} \cdot \frac{\rho-\rho_{0}}{\rho_{\text {etd }}-\rho_{0}}$.

\subsection{Initial data}

When carrying out the numerical studies of the ignition process, the thermophysical characteristics have been used (Table 1).

As it has been indicated earlier, the heating of the wood proceeds under conditions of its thermal decomposition, accompanied by the release of the gaseous, solid and liquid reaction products. The composition of the gaseous pyrolysis products has been shown in Table 2.

The thermochemical constants have been given in Table 3. The numerical studies have been carried out with the following initial data: $\mathrm{T}_{0}=\mathrm{T}(\mathrm{r}, \theta, 0)=298 \mathrm{~K} p_{0}=101325 \Pi$ П; $\eta_{0}=0 ; \mu=0,018 \mathrm{~kg} /$ mol; $K_{P}=10^{-11} \mathrm{~m}^{2}$.
Table 2

Chemical composition of the pyrolysis products (according to the data of [47]).

\begin{tabular}{ll}
\hline Name & Content by weight,\% \\
\hline $\mathrm{CO}_{2}$ & 14,0 \\
$\mathrm{CO}$ & 39,3 \\
$\mathrm{CH}_{4}$ & 5,96 \\
$\mathrm{H}_{2}$ & 1,07 \\
\hline
\end{tabular}

Table 3

Thermochemical constants.

\begin{tabular}{llll}
\hline Reaction & $\begin{array}{l}\text { The activation energy } E_{i} \\
J /(\mathrm{mol})\end{array}$ & \multicolumn{2}{l}{$\begin{array}{l}\text { Preexponent of } \\
\text { reaction } k_{i}(1 / \mathrm{s})\end{array}$} \\
\hline $\begin{array}{l}\text { Thermal decomposition } \\
w \rightarrow \text { gas }\end{array}$ & $88.6 \cdot 10^{3}$ & $1.43 \cdot 10^{4}$ & {$[48]$} \\
$w \rightarrow$ char & $106.5 \cdot 10^{3}$ & & \\
$w \rightarrow$ tar & $112.7 \cdot 10^{3}$ & $7.4 \cdot 10^{6}$ & \\
$\mathrm{H}_{2} \mathrm{O}+\mathrm{C}=\mathrm{CO}+\mathrm{H}_{2}$ & $220 \cdot 10^{3}$ & $4.12 \cdot 10^{6}$ & \\
$\mathrm{CO}+0.5 \cdot \mathrm{O}_{2}=\mathrm{CO}_{2}$ & $200.0 \cdot 10^{3}$ & $2.07 \cdot 10^{7}$ & {$[49]$} \\
$\mathrm{H}_{2}+0.5 \cdot \mathrm{O}_{2}=\mathrm{H}_{2} \mathrm{O}$ & $105.0 \cdot 10^{3}$ & $2.50 \cdot 10^{12}$ & {$[50]$} \\
$\mathrm{CH}_{4}+\mathrm{O}_{2}=\mathrm{CO}_{2}+2 \cdot \mathrm{H}_{2} \mathrm{O}$ & $35 \cdot 10^{3}$ & $1.13 \cdot 10^{17}$ & {$[51]$} \\
& & $5.1 \cdot 10^{14}$ & {$[52]$} \\
\hline
\end{tabular}

\subsection{The method of solving equations}

The formulated problem of mathematical physics has been solved by the finite difference method [53]. The equations approximation has been carried out with using an implicit fourpoint difference template (Fig. 6.) [54]. For example, equation (1) in the Cartesian coordinate system has been approximated as follows:

$$
\begin{aligned}
\frac{\partial H_{w}}{\partial t}+C_{s} \rho_{s}\left(U_{s} \frac{\partial T}{\partial x}+V_{s} \frac{\partial T}{\partial y}\right)= & \frac{\partial}{\partial x}\left(\lambda_{w} \frac{\partial T}{\partial x}\right)+\frac{\partial}{\partial y}\left(\lambda_{w} \frac{\partial T}{\partial y}\right) \\
& -\frac{Q_{\text {eva }} W_{\text {eva }}}{h_{\text {spr }}} \delta\left(\xi_{\text {eva }}\right) \\
& -\sum_{i=1}^{4} Q_{i} W_{i} \Phi\left(\xi_{\text {eva }}\right)
\end{aligned}
$$

$$
\Rightarrow\left\{\begin{array}{l}
\frac{H_{i, j}^{n+1 / 2}-H_{i, j}^{n}}{\tau}+C_{s} \rho_{s} U_{i, j} \frac{T_{i+1, j}^{n+1 / 2}-T_{i-1, j}^{n+1 / 2}}{2 h_{x}} \Phi\left(\xi_{\text {eva }}\right)=\frac{1}{h_{x}}\left(\lambda_{i+1 / 2, j} \frac{\left(T_{i+1, j}^{n+1 / 2}-T_{i, j}^{n+1 / 2}\right)}{h_{x}}-\lambda_{i+1 / 2, j} \frac{\left(T_{i+1, j}^{n+1 / 2}-T_{i, j}^{n+1 / 2}\right)}{h_{x}}\right)- \\
-\frac{Q_{\text {eva }} W_{\text {eva }}}{h_{\text {spr }}} \delta\left(\xi_{\text {eva }}\right)-\frac{\sum_{i=1}^{4} Q_{i} \cdot W_{i}}{2}
\end{array}\right.
$$




$$
\Rightarrow\left\{\begin{array}{l}
\frac{H_{i, j}^{n+1 / 2}-H_{i, j}^{n}}{\tau}+C_{s} \rho_{s} V_{s} \frac{T_{i, j+1}^{n+1}-T_{i, j-1}^{n+1}}{h_{y}} \Phi\left(\xi_{\text {eva }}\right)=\frac{1}{h_{y}}\left(\lambda_{i, j+1 / 2} \frac{\left(T_{i, j+1}^{n+1}-T_{i, j}^{n+1}\right)}{h_{y}}-\lambda_{i, j-1 / 2} \frac{\left(T_{i, j}^{n+1}-T_{i, j-1}^{n+1}\right)}{h_{y}}\right)- \\
-\frac{Q_{\text {eva }} W_{\text {eva }} \delta\left(\xi_{\text {eva }}\right)-\frac{\sum_{i=1}^{4} Q_{i} \cdot W_{i}}{2}}{h_{\text {spr }}}
\end{array}\right.
$$

The thermal conductivity approximation $\lambda$ is carried out according to the scheme:

$$
\lambda_{i+1 / 2, j}=0.5 \cdot\left(\lambda_{i+1, j}+\lambda_{i, j}\right), \lambda_{i-1 / 2, j}=0.5 \cdot\left(\lambda_{i-1, j}+\lambda_{i, j}\right)
$$

Equations (2)-(7) have been approximated by a similar scheme. It should be noted that the calculations of the fuel ignition during the intensive phase transformations (in particular evaporation) are among the most difficult in the combustion theory. The latter is due to the fact that the evaporation of water flows in a very narrow (much smaller than the linear size of the particle) area (the front of evaporation). In this case, as a rule, the thermal effects of the phase transitions are very high (up to $2.5 \cdot 10^{6} \mathrm{~J} / \mathrm{kg}$ ). As a result, an internal flow of heat emerges in the wet particle and a substantially inhomogeneous heterogeneous structure is formed. It should be noted that the asymptotics of the processes of evaporation and ignition are significantly different. A direct ignition (a chemical reaction) is a quick process, evaporation is slow. Such a correlation of the characteristic times substantially complicates the theoretical studies of the combustion initiation processes. It should also be noted that mathematical modeling of the phase transition processes (evaporation of water) in a multidimensional formulation (2-dimensional or 3-dimensional) is a very difficult operation, especially for the bodies with a complex geometry that does not have the properties of an axial symmetry. This is due to the fact that during the heating process, the front of the phase transition is formed in the body, the boundary of which can take various forms, depending on the heat

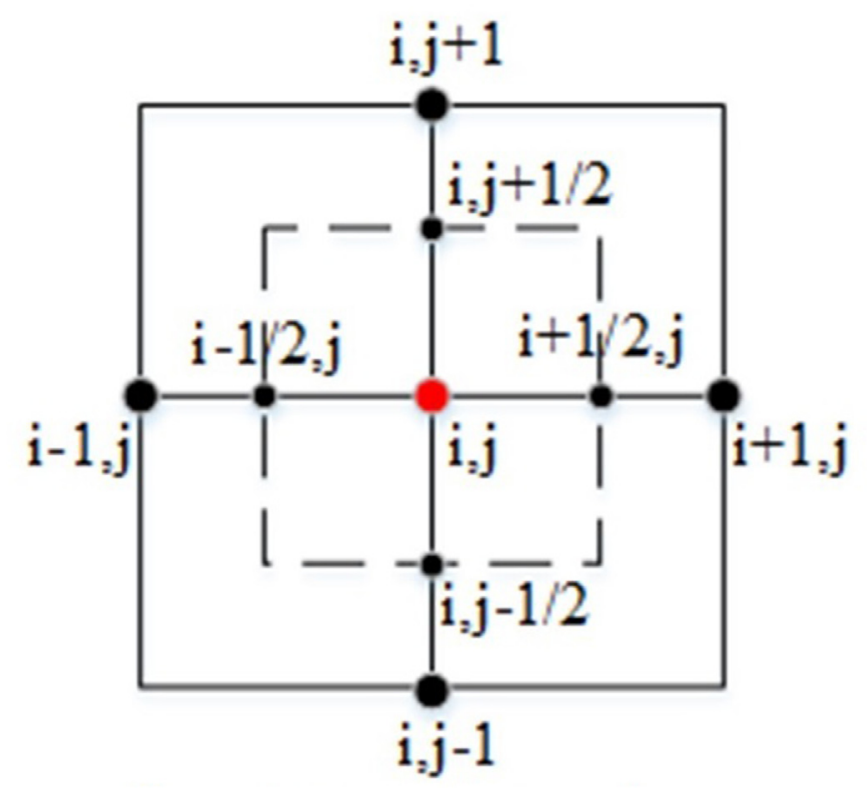

Fig. 6. Approximation scheme. fluxes in the vicinity of the phase transition zone. Such tasks are called the Stefan monofront problems. The latter problems are some of the most difficult in mathematical physics. For this reason, a special algorithm based on the method of the implicit separation of the phase transition front has been developed to solve system (1)-(7) [55].

\section{Results and discussions}

In order to verify the mathematical models (1)-(7), a comparative analysis of the experimental and calculated values of the delay times for the ignition of the wood particles in the form of a cylinder and a cube has been made. Fig. 7 shows the experimental and theoretical dependences of the ignition delay times of a particle of (two typical forms: a cylinder and a cube) of wet wood from the temperature of the external environment. The characteristic particles size is $\sim 3 \mathrm{~mm}$. The analysis of these dependences shows that the form of the particle has a significant (up to $10 \%$ of the delay in the ignition delay) effect on the characteristics and conditions of ignition of the wood. It can be noted that cylindrical wood particles begin to burn earlier than cubic ones. It should be noted that the dependences $t_{i g n}=f(T)$ have an exponential character. The latter is due to the significant influence of the processes of the thermal preparation (radiation-convective heating, evaporation of water, pyrolysis of solid fuel) and ignition (inflammation of volatiles).

Fig. 8 shows the experimental and theoretical dependences of the ignition delay times on the temperature of the oxidizer. The characteristic particles size of woody biomass is $\sim 1 \mathrm{~mm}$. By the

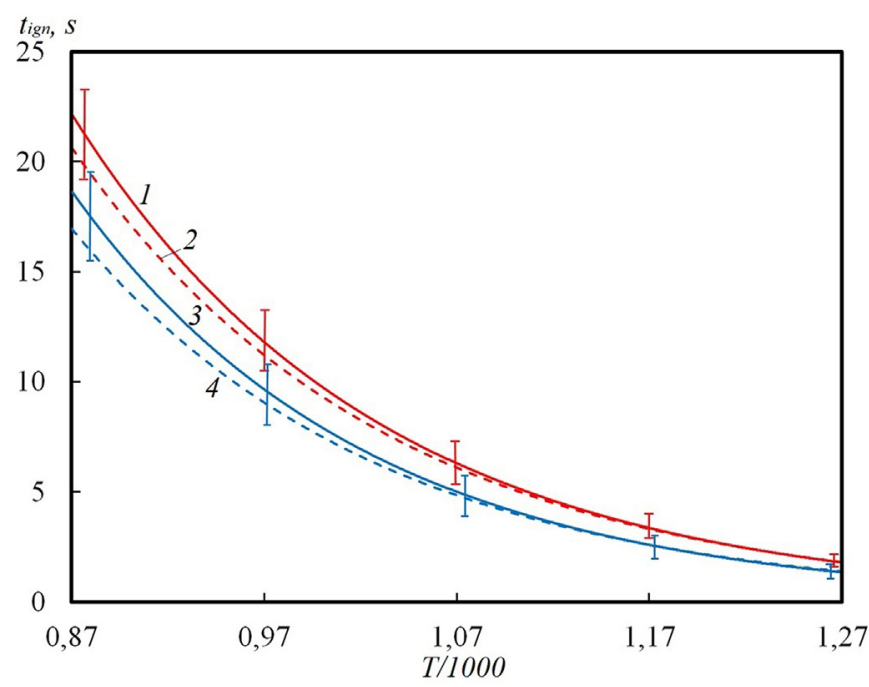

Fig. 7. Dependence of the time delay of ignition of the wood particles a cubic (curves 1 , 2 ) and a cylindrical $(3,4)$ form on the temperature of the external environment. The characteristic particle size is $\delta=3 \cdot 10^{-3} \mathrm{~mm} .1,3$ - Experiment; 2,4 is the solution of system (1)-(7). 


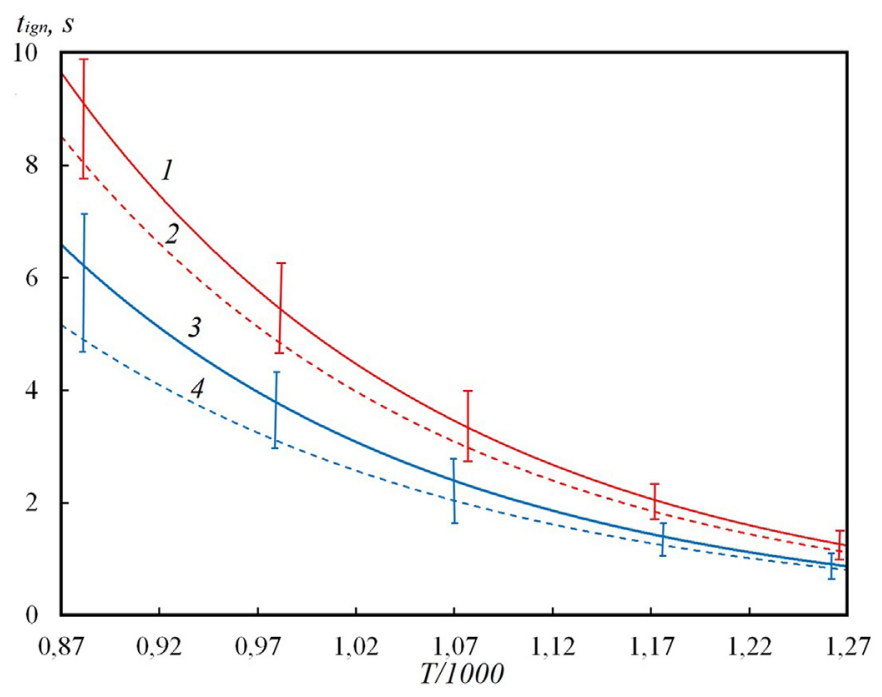

Fig. 8. Dependence of the delay time of ignition of the wood particles cubic (curves 1, 2 ) and cylindrical $(3,4)$ forms on the temperature of the external environment. The characteristic particle size is $\delta=1 \cdot 10^{-3} \mathrm{~mm}$. 1, 3 - Experiment; 2,4 is the solution of system (1)-(7).

results of the analysis in Fig. 8, it is possible to draw the conclusions analogous to Fig. 7.

The comparative analysis of the theoretical and experimental values of the ignition delay times shows their good accordance. It can be concluded that the developed mathematical model of the particles inflammation of wet wood biomass has a rather high predictive potential. After verification of the mathematical model, modeling of the processes of the wet wood particles ignition of three (a sphere, a cylinder and a cube) of various forms has been carried out. It should be noted that in order to carry out a comparative analysis of the dynamics of the wood particles ignition of various forms, it is necessary to choose a criterion for estimating the comparison of the particles. The use of a characteristic particle size for this purpose is not sufficiently justified. This is due primarily to the fact that the geometry of the particles is significantly different, and the cubic particles with ribs $\approx 3 \mathrm{~mm}$ exceed in volume and mass the cylindrical and spherical particles with a diameter of $\approx 3 \mathrm{~mm}$. For this reason, the main criterion for

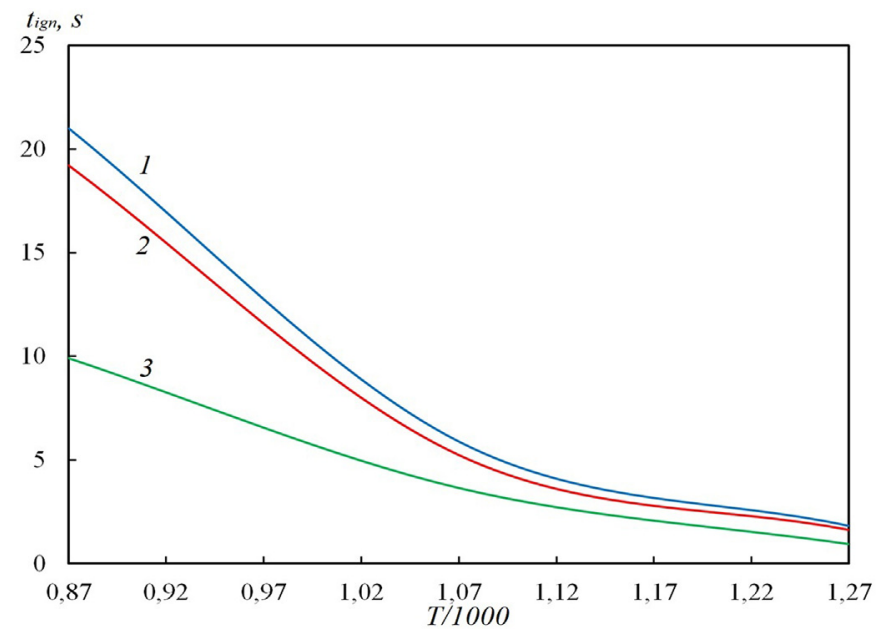

Fig. 9. Dependence of the delay time of the wet wood particles ignition of various forms if the masses are identical. $1-$ a particle of wood in the form of a cube; $2-a$ cylinder; 3 - a sphere. comparison is the mass of the particle:

$m_{k u b}=m_{c i l}=m_{\text {sph }}$

In this case, it is possible to calculate the ratios of the characteristic particle sizes of the cubic, spherical and cylindrical forms:

$m_{i}=\delta^{3} \cdot \rho_{w}=\pi \cdot r_{\text {out }}^{2} \cdot \delta \cdot \rho_{w}=\frac{4}{3} \cdot \pi \cdot r_{\text {out }}^{3} \cdot \rho_{w}$

Fig. 9 shows the values of the ignition delay times for the wet wood particles of three forms (a cube, a cylinder and a sphere), provided that the masses are equal. The comparative analysis of the dependencies (Fig. 9) shows that the wood particles in the form of a sphere ignite at $870 \mathrm{~K} \leq \mathrm{T}_{\mathrm{g}} \leq 1270 \mathrm{~K}$ much faster than cylindrical and cubic ones. This is due to the fact that, as is known from the theory of heat transfer [20], bodies in the form of a sphere are heated much faster than cylindrical and cubic bodies. Accordingly, the main stages (evaporation of water, thermal decomposition, release of volatiles) of the thermal preparation of fuel for ignition also proceed faster. In this case, we can say that from the point of view of the dynamics of ignition, the wood particles in the form of a sphere are most advantageous in the preparation of fuel for combustion.

In order to justify the above-mentioned thesis on the significantly higher dynamics of the process of heating the fuel layer upon ignition of the spherical particles, in comparison with cylindrical and cubic ones, we'll introduce the heating rate function:

$\Psi=\frac{d \bar{T}}{d t}$

Where:

$\bar{T}=\frac{1}{V} \oiint_{V} T(r, \theta, \varphi, t) d V$

Fig. 10 shows the function dependences of the rate of heating of the fuel particle on time at the ambient temperature. It can be noted that spherical particles are heated much more intensively compared with cylindrical and cubic particles, with taking into account the identity of their masses. This is due to the geometry of the particles.

Fig. 11 shows the delay times of ignition of the particles char residue of biomass of three forms (a cube, a cylinder and a sphere). The analysis of the dependences $t_{i g n}\left(T_{g}\right)$ shows that char begins to

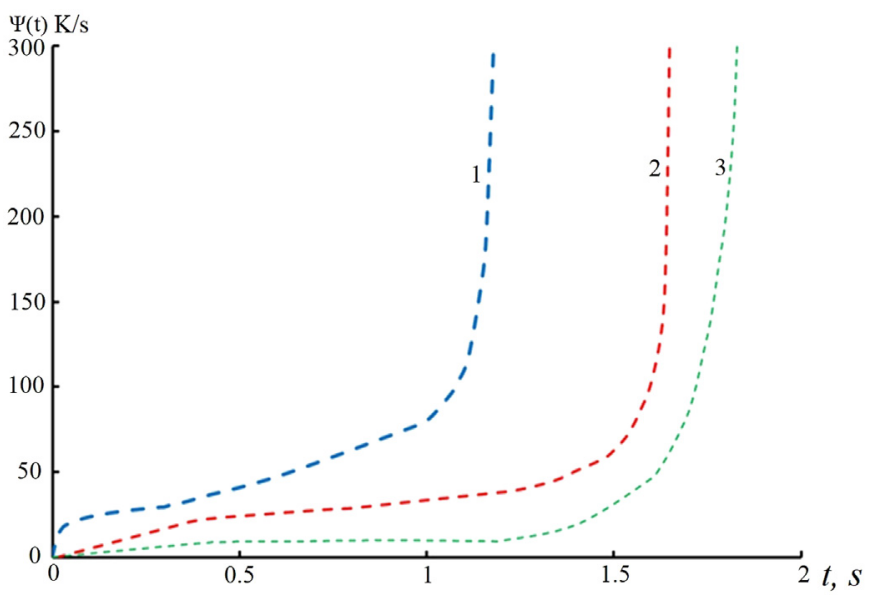

Fig. 10. Dependence of the heating rate of wood particles of three different forms: 1 - a particle of wood in the form of spheres; 2 - the cylinder; 3 - cube. 


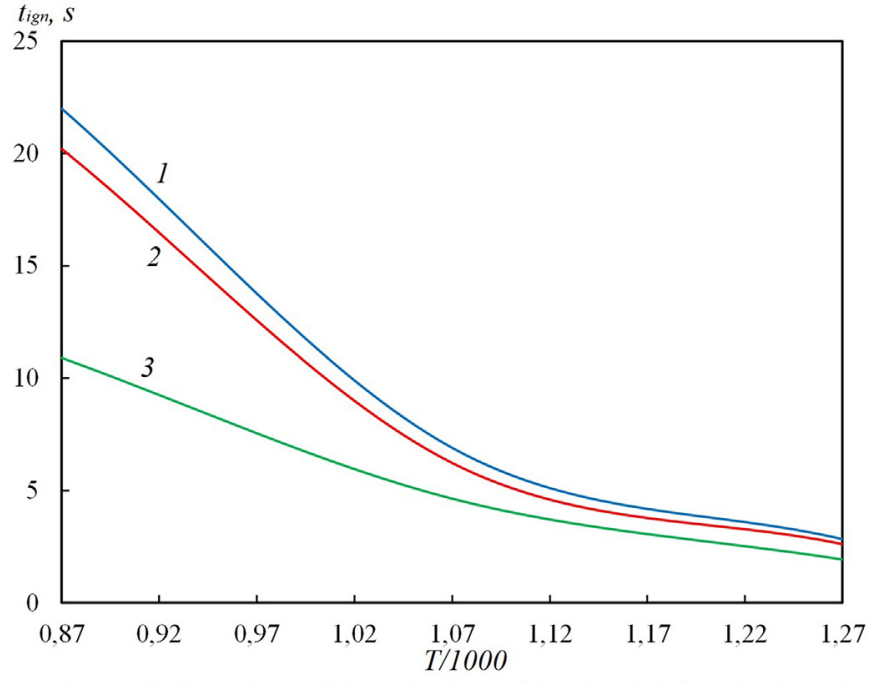

Fig. 11. Dependence of the delay time of the char ignition of wet wood particles of various forms provided that the masses are identical.

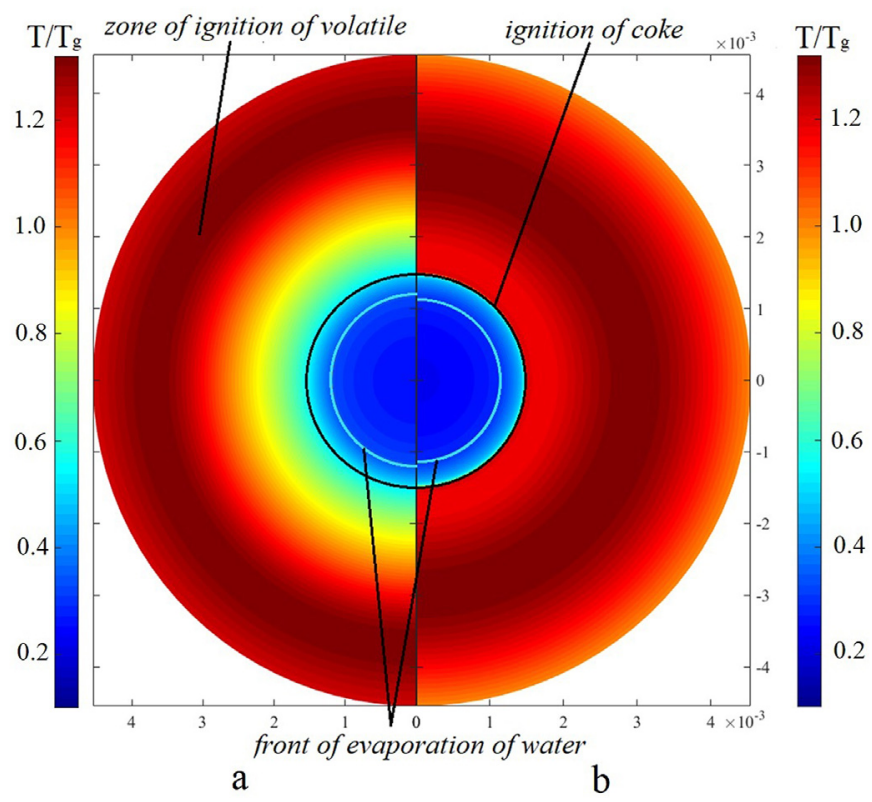

Fig. 12. Temperature fields at the moment of ignition of volatiles (a) and char (b) when a particle of the wet wood biomass of a cylindrical form is heated in a hightemperature medium.

burn almost immediately after ignition of the gaseous pyrolysis products. The interval between the instants of time of ignition of pyrolysis gases and char does not exceed $1.0 \mathrm{~s}$. This is due to the fact that in the conditions of burning of volatiles heat flow to the surface of the particle increases substantially. As a result, the combustion process of the char fuel residue is initiated.

1 - a particle of wood in the form of a cube; $2-$ a cylinder; $3-$ a sphere.

Fig. 12 (a, b) shows the numerically calculated temperature fields at the instants of ignition of gaseous pyrolysis products (a) and char ignition (b) during heating of wet wood particles of a cylindrical form (diameter $\delta=3 \times 10^{-3} \mathrm{~m}$, ambient temperature $\mathrm{T}_{\mathrm{g}}=1270 \mathrm{~K}$ ). It is clearly seen that gas-phase ignition occurs at the outer boundary of the gas rea. The numerical simulation has

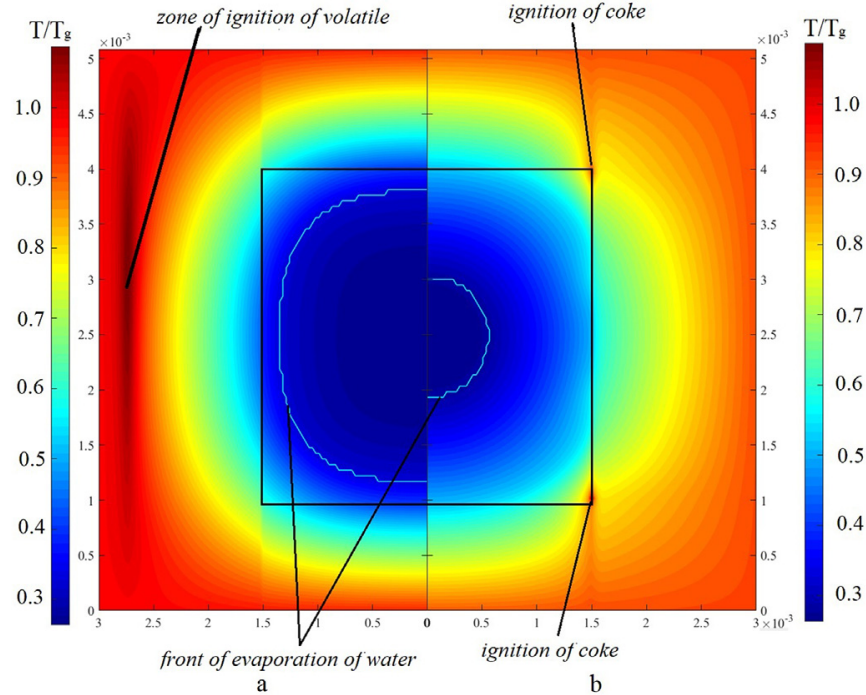

Fig. 13. Temperature fields at the moment of ignition of volatiles (a) and char (b) when a particle of the wet wood biomass of a cubic form is heated in a high-temperature medium.

showed that the particles with a characteristic size of $3 \cdot 10^{-3} \mathrm{~m}$ are ignited until they are completely dehydrated. It is obvious that the mechanism of burning of such wood under conditions of continuous evaporation differs significantly from the oxidation of previously dried biomass. Water vapors are blown into the boundary layer of the particle and, accordingly, increase the reaction zone of pyrolysis products. Ignition of char flows on the surface of the particle. It can be noted that the initiation of heterogeneous combustion occurs before the complete evaporation of water in the deep layers of wood.

Fig. 13 (a, b) shows the temperature fields at the moment of ignition of a cubic particle of wood (with the characteristic size $\delta=$ $3 \cdot 10^{-3} \mathrm{~m}$ ). It can be noted that the ignition of gaseous pyrolysis products (Fig. 13 a) occurs at a distance of about $1.0 \cdot 10^{-3} \mathrm{~m}$ from the surface of the particle. Fig. 13 (a, b) shows the temperature fields at the moment of ignition of a cubic particle of wood (with the characteristic size $\delta=3 \cdot 10^{-3} \mathrm{~m}$ ). It can be noted that the ignition of gaseous pyrolysis products (Fig. 13 a) occurs at a distance of about $1.0 \cdot 10^{-3} \mathrm{~m}$ from the surface of the particle. Accordingly, it is possible to draw a conclusion similar to Fig. 12 about the formation of a buffer zone in the boundary layer by water vapors. It can be noted that the ignition of char is localized at the corners of the particle. This is due to the fact that when warming up at the corner points of the cube intense heat flows are concentrated.

As the ignition of volatiles (a) and char (b) occurs before the complete evaporation of water in the porous structure of wood, then a nonlinear evaporation front is formed. The latter factor leads to a substantial inhomogeneity of the temperature field. Accordingly, it can reasonably be assumed that the application of the zerodimensional mathematical models of ignition (by analogy with [25]) is unreasonable.

Fig. 14 (a, b) shows the temperature fields in the "particle - gas medium" system at the moment of ignition of volatiles (a) and char (b) with heating of the spherical particles (diameter $\delta=3 \cdot 10^{-3} \mathrm{~m}$ ) of wet wood biomass. By analogy with Figs. 12 and 13, it can be noted that the initiation of combustion takes place in the outer gas area at a distance of $1-1.5 \times 10^{-3} \mathrm{~m}$ from the surface of the particle. In this case, the ignition of both gaseous pyrolysis products and char takes place even before the moisture evaporates completely.

The analysis of Figs. 12-14 shows that the formation of a dry 


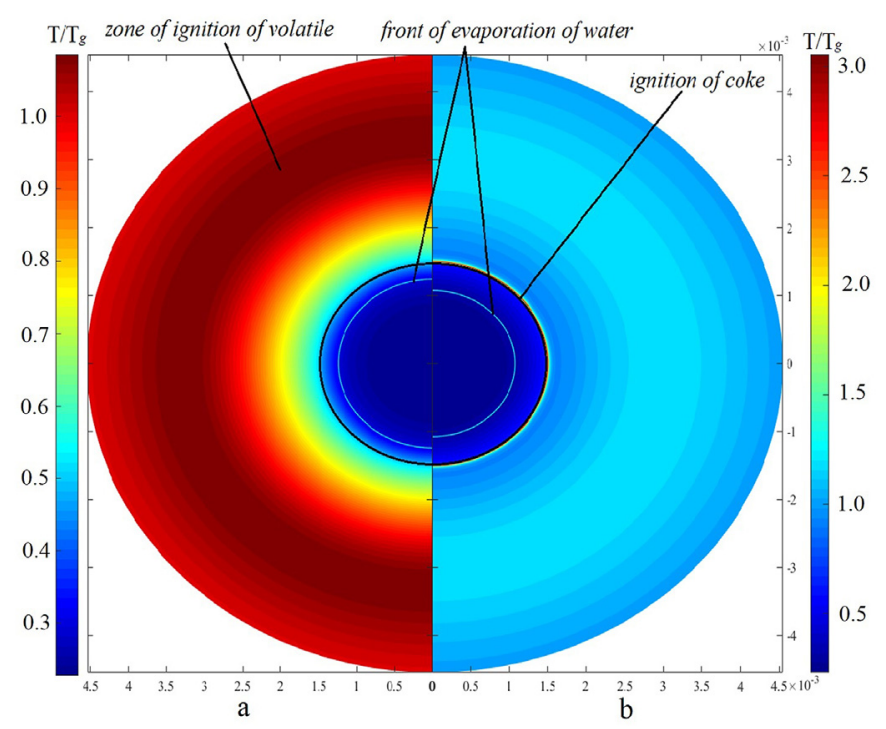

Fig. 14. Temperature fields at the moment of ignition of volatile (a) and coke (b) when a particle of moist wood biomass is heated in a spherical shape in a high-temperature environment.

pyrolyzed layer is necessary to ignite a wood particle. In the case of the particles ignition of a spherical form, the dimensions of this layer are smaller than when there is ignition of cylindrical or cubic particles.

\section{Analysis of the possibility of the practical application of the research results}

\subsection{Dusty burning of wood biomass in the boiler units}

One of the most common ways of burning wood is burning it in the form of a finely dispersed pseudo-liquefied dusty air mixture in the furnace device of boiler units (by analogy with [56]). This method is the most convenient from the point of view of the organization of the combustion process and the supply of fuel to the combustion space of the boiler unit. In this case, the air-fuel mixture is blown into the boiler furnace, where it ignites and burns.

The characteristics of ignition and combustion of a fuel torch (a large group of wood particles) according to the theory of combustion [31] of the solid fuels are caused (primarily) by the burning behavior of a single particle. Such a particle is called a particle marker (or a typical particle) [57]. It is worth noting that when the wood-air mixture is sprayed, a polydisperse stream of wood particles is formed [58], which differ significantly in form and size. In this case, the particles are in the form of, as a rule, irregular polyhedral with a large number of edges and corners. As it has been previously established [29], the form of the wood particle can influence the characteristics of its heating and thermal decomposition. It is practically impossible to investigate the influence of the whole possible variety of forms and sizes of the wood particles on the characteristics of their ignition (neither in experiments nor theoretically). For this reason, the most expedient is to study the processes of ignition of the wood particles (under conditions of their high-temperature heating by an oxidizing medium) of the most typical forms: a cube, a cylinder, a sphere. Such forms are homeomorphic to the forms of the wood particles (irregular polyhedral) that are formed during spraying. Accordingly, it can be said that according to the results of the conducted researches, the values of the delay time of ignition of the wood particles have been obtained, which are extreme (upper (cube) and lower (sphere)) estimates of $t_{i g n}$ values under conditions of heating of the wood particles in the stream of the high-temperature environment. The latter will allow us to describe the temperature fields of the fuel torch for various forms of the fuel particles.

\subsection{Biomass burning in a fixed fluidized bed}

One of the most promising methods for the energy use of biomass is the technology of its combustion in the form of pellets in the furnaces of the water boilers of the local heat supply systems [59]. At the same time, the technological cycle [60] allows to produce pellets of various forms (cylinders, parallelepipeds, cubes, spheres, etc.) and sizes. The latter is determined by the type of molding cells used for the pelletizer [61]. Accordingly, it is possible to carry out a procedure of optimizing the form of the fuel particles in order to reduce the induction and oxidation periods and, accordingly, to increase the efficiency of burning wood. The analysis of the results of the research shows that the particles in the form of a sphere have the best (judging by the values of the ignition delay time) burning characteristics. The spherical particles of biomass faster than others (cylindrical and cubic) ignite and, accordingly, burn. Also, the results of [62] show that the spherical particles of biomass are more (than, for example, cylinders) compactly arranged in a layer, provided that the particles are sufficiently well blown off with an oxidizing agent.

But it should be said that the production of the spherical particles is quite expensive (we need special-formed pelletizers [23]). For this reason, it can be reasonably concluded that the choice of the base pellets (for a boiler unit) of wood pellets of one form or another (a sphere, a cylinder, a cube) is a complex optimization task of the technical and engineering study, which is solved at the stage of the experimental development.

\section{Conclusion}

According to the results of the experimental and theoretical studies, a significant effect of the form of a wet wood particle on the characteristics of its ignition has been established. The experimental studies of the ignition processes have been carried out for fuel particles of two different forms: a cube and a cylinder. By the results of the experiments it has been established that fuel particles of a cylindrical form with comparable dimensions are ignited faster than cubic ones.

According to the results of the theoretical studies, a new mathematical model of the process of the particles ignition of wet wood biomass has been developed. It takes into account the joint course of the main stages of the thermal preparation under conditions of intensive phase (evaporation of water with taking into account the nonequilibrium parameters on the boundary of the phase transition front) the thermochemical (pyrolysis of fuel with taking into account the complex kinetics of the process) transformations. The comparative analysis of the delay times obtained experimentally and theoretically has showed their good accordance. The latter testifies to the high predictive potential of the developed mathematical model of the ignition process.

The comparative analysis (based on the results of modeling) has showed that, under the condition that the masses of fuel particles are comparable; the spherical ones are ignited faster than cubic and cylindrical ones. The slowest, the particles in the form of a cube are inflamed. The latter is confirmed by the analysis of the functions of the heating rates of the fuel particles of three forms. Spheres are heated much faster than cylinders and cubes. The latter is due to the geometry of the bodies. 


\section{Acknowledgments}

This research was performed within the framework of the strategic plan for the development of National Research Tomsk Polytechnic University as one of the world-leading universities within framework Russian State Project “Science" 8.13264.2018/8.9 (project VIU- SEC I.N. Butakov - 203/2019).

\section{Appendix A. Supplementary data}

Supplementary data to this article can be found online at https://doi.org/10.1016/j.renene.2019.04.046.

\section{References}

[1] A. Favero, E. Massetti, Trade of woody biomass for electricity generation under climate mitigation policy, Resour. Energy Econ. 36 (2014) 166-190.

[2] R.S. Dodder, P.O. Kaplan, A. Elobeid, S. Tokgoz, S. Secchi, L.A. Kurkalova, Impact of energy prices and cellulosic biomass supply on agriculture, energy, and the environment: an integrated modeling approach, Energy Econ. 51 (2015 77-87.

[3] L. Boying, C.R. Shumway, J.K. Yoder, Lifecycle economic analysis of biofuels: accounting for economic substitution in policy assessment, Energy Econ. 67 (2017) 146-158.

[4] V. Bertrand, Carbon and energy prices under uncertainty: a theoretical analysis of fuel switching with heterogenous power plants, Resour. Energy Econ. 38 (2014) 198-220.

[5] G. Giannoccaro, B.C. de Gennaro, E. De Meo, M. Prosperi, Assessing farmers' willingness to supply biomass as energy feedstock: cereal straw in Apulia (Italy), Energy Econ. 61 (2017) 179-185.

[6] Y. Voytenko, P. Peck, Organisational frameworks for straw-based energy systems in Sweden and Denmark, Biomass Bioenergy 38 (2012) 34-48.

[7] Lixia He, Burton C. English, Robert J. Menard, Dayton M. Lambert, Regional woody biomass supply and economic impacts from harvesting in the southern U.S, Energy Econ. 60 (2016) 151-161.

[8] André F.P. Lucena, Leon Clarke, Roberto Schaeffer, Szklo Alexandre, R. Pedro, R. Rochedo, Larissa P.P. Nogueira, Kathryn Daenzer, Angelo Gurgel Alban Kitous, Tom Kober, Climate policy scenarios in Brazil: a multi-model comparison for energy, Energy Econ. 56 (2016) 564-574.

[9] Aparna Sawhney, M. Rahul, Examining the regional pattern of renewable energy CDM power projects in India, Energy Econ. 42 (2014) 240-247.

[10] Y. Voytenko, P. Peck, Organisational frameworks for straw-based energy systems in Sweden and Denmark, Biomass Bioenergy 38 (2012) 34-38.

[11] S.V. Vassilev, C.G. Vassileva, V.S. Vassilev, Advantages and disadvantages of composition and properties of biomass in comparison with coal: an overview, Fuel 158 (2015) 330-350.

[12] K.C. Akyu, Y. Balaban, Wood fuel trade in European Union, Biomass Bioenergy 35 (2011) 1588-1599.

[13] G. Wielgosinski, P. Łechtanska, O. Namiecinska, Emission of some pollutants from biomass combustion in comparison to hard coal combustion, J. Energy Inst. 90 (2017) 787-796.

[14] Weidong Fan, Li Yu, Qinghong Guo, Can Chen, Yong Wang, Coal-nitrogen release and NOx evolution in the oxidant-staged combustion of coal, Energy 125 (2017) 417-426.

[15] C.A. Garcia, G. Hora, State-of-the-art of waste wood supply chain in Germany and selected European countries, Waste Manag. 70 (2017) 189-197.

[16] S. Nishiguchi, T. Tabata, Assessment of social, economic, and environmental aspects of woody biomass energy utilization: direct burning and wood pellets, Renew. Sustain. Energy Rev. 57 (2016) 1279-1286.

[17] W. Simpson, A. TenWolde, Physical Properties and Moisture Relations of Wood. Ood Handbook: Wood as an Engineering Material, USDA Forest Service, Forest Products Laboratory, Madison, W.I, 1999. General technical report FPL; GTR-113: Pages 3.1-3.24.

[18] J. Silva, J. Teixeira, S. Teixeira, S. Preziati, J. Cassiano, CFD modeling of combustion in biomass furnace, Energy Procedia 120 (2017) 665-672.

[19] S. Mani, L.G. Tabil, S. Sokhansanj, Effects of compressive force, particle size and moisture content on mechanical properties of biomass pellets from grasses, Biomass Bioenergy 30 (2006) 648-654.

[20] H. Carslaw, J. Jaeger, Conduction of Heat in Solids, 2 edition, Oxford University Press, USA, 1959, p. 510.

[21] D. Magalhães, F. Kazanç, A. Ferreira, M. Rabaçal, M. Costa, Ignition behavior of Turkish biomass and lignite fuels at low and high heating rates, Fuel 207 (2017) 154-164.

[22] J.M. Jones, A. Saddawi, B. Dooley, E.J.S. Mitchell, J. Werner, D.J. Waldron, S. Weatherstone, A. Williams, Low temperature ignition of biomass, Fuel Process. Technol. 134 (2015) 372-377.

[23] A. Kijo-Kleczkowska, K. Środa, M. Kosowska-Golachowska, T. Musiał K. Wolski, Combustion of pelleted sewage sludge with reference to coal and biomass, Fuel 170 (2016) 141-160.

[24] S.G. Smith, Effects of Moisture on Combustion Characteristics of Live California
Chaparral and Utah. Foliage, M.S., Chemical Engineering, Brigham Young University, USA, 2005.

[25] M.J. DiDomizio, P. Mulherin, E.J. Weckman, Ignition of wood under timevarying radiant exposures, Fire Saf. J. 82 (2016) 131-144.

[26] B.L. Yashwanth, B. Shotorban, S. Mahalingam, C.W. Lautenberger, D.R. Weise, A numerical investigation of the influence of radiation and moisture content on pyrolysis and ignition of a leaf-like fuel element, Combust. Flame 163 (2016) 301-316.

[27] V.A. Shargatov, A.T. Il'ichev, G.G. Tsypkin, Dynamics and stability of moving fronts of water evaporation in a porous medium, Int. J. Heat Mass Transf. 83 (2015) 552-561.

[28] Hong Lu, Elvin Ip, Justin Scott, P. Foster, M. Vickers, L.L. Baxter, Effects of particle shape and size on devolatilization of biomass particle, Fuel 89 (2010) 1156-1168.

[29] R. Mehrabian, S. Zahirovic, R. Scharler, I. Obernberger, S. Kleditzsch, S. Wirtz, V. Scherer, Hong Lu, L.L. Baxter, A CFD model for thermal conversion of thermally thick biomass particles, Fuel Process. Technol. 95 (2012) 96-108.

[30] Y. Chen, K. Aanjaneya, A. Atreya, A study to investigate pyrolysis of wood particles of various shapes and sizes, Fire Saf. J. 91 (2017) 820-827.

[31] D.B. Spalding, Combustion and Mass Transfer, Elsevier, 1978.

[32] V.N. Vilynov, Theory of Ignition of Condensed Materials, 1984. Novosibirsk.

[33] D.A. Frank-Kamenetskii, Diffusion and Heat Exchange in Chemical Kinetics, Princeton University Press, 2015.

[34] H. Rezaei, C. Jim Lim, A. Lau, S. Sokhansanj, Size, shape and flow characterization of ground wood chip and ground wood pellet particles, Powder Technol. 301 (2016) 737-746.

[35] É. Trudel, W.L.H. Hallett, E. Wiens, J.D. O'Neil, M.K. Busigin, D. Berdusco, Fuel particle shape effects in the packed bed combustion of wood, Combust. Flame 198 (2018) 100-111.

[36] S.V. Syrodoy, G.V. Kuznetsov, N.Y. Gutareva, K.A. Bugaeva, R.I. Taburchinov, The conditions and characteristics of wood particles ignition in the stream of the high temperature gases, Combust. Sci. Technol. 190 (2018) 663-686.

[37] J. Wolberg, Data Analysis Using the Method of Least Squares: Extracting the Most Information from Experiments, Springer-Verlag, Berlin, 2006, p. 264.

[38] D.O. Glushkov, G.V. Kuznetsov, P.A. Strizhak, S.V. Syrodoy, A mathematical model for processes in coal-water slurries containing petrochemicals under heating energy and Fuels 32 (8) (16 August 2018) 8789-8802.

[39] D.O. Glushkov, G.V. Kuznetsov, P.A. Strizhak, S.V. Syrodoy, Mathematical model simulating the ignition of a droplet of coal water slurry containing petrochemicals, Energy 150 (1 May 2018) 262-275.

[40] S.V. Syrodoy, The influence of radiative-convective heat transfer on ignition of the drops of coal-water fuel, Thermophys. Aeromechanics 25 (3) (1 May 2018) 429-443.

[41] S.V. Syrodoi, G.V. Kuznetsov, V.V. Salomatov, Effect of the shape of particles on the characteristics of the ignition of coal-water fuel, Solid Fuel Chem. 49 (Issue 6) (1 November 2015) 365-371.

[42] Hu Luo, Liwei Bao, Lingzhao Kong, Yuhan Sun, Low temperature microwaveassisted pyrolysis of wood sawdust for phenolic rich compounds: kinetics and dielectric properties analysis, Bioresour. Technol. 238 (2017) 109-115.

[43] Colomba Di Blasi, Modeling chemical and physical processes of wood and biomass pyrolysis, Prog. Energy Combust. Sci. 34 (2008) 47-90.

[44] P. Atreya, Y. Olszewski, H. Chen, R. Baum, The effect of size, shape and pyrolysis conditions on the thermal decomposition of wood particles and firebrands, Int. J. Heat Mass Transf. 107 (2017) 319-328.

[45] T.G. Shendrik, Y.V. Tamarkina, T.V. Khabarova, V.A. Kucherenko, N.V. Chesnokov, B.N. Kuznetsov, Formation of the pore structure of brown coal upon thermolysis with potassium hydroxide, Solid Fuel Chem. 43 (2009) 51.

[46] S. Lagüela, P. Bison, F. Peron, P. Romagnoni, Thermal conductivity measurements on wood materials with transient plane source technique, Thermochem. Acta 600 (2015) 45-51.

[47] A.I. Moreno, R. Font, Pyrolysis of furniture wood waste: decomposition and gases evolved, J. Anal. Appl. Pyrolysis 113 (2015) 464-473.

[48] F. Thurner, U. Mann, Kinetic investigation of wood pyrolysis, Ind. Eng. Chem. Process Des. Dev. 20 (1981) 482-488.

[49] K. Umeki, K. Yamamoto, T. Namioka, K. Yoshikawa, High temperature steamonly gasification of woody biomass, Appl. Energy 87 (2010) 791-798.

[50] J. Mantzaras, Catalytic combustion of syngas, Combust. Sci. Technol. 180 (2008) 1137-1168.

[51] Chang Jian Wang, J. Wen, Shou Xiang Lu, Guo Jin, Single-step chemistry model and transport coefficient model for hydrogen combustion, Sci. China Technol. Sci. 55 (2012) 2163-2168.

[52] Xin Zhang, Tao Wang, Jian Xu, Shizhuo Zheng, Xiaosen Hou, Study on flamevortex interaction in a spark ignition engine fueled with methane/carbon dioxide gases, J. Energy Inst. 91 (2018) 133-144.

[53] P.J. Roache, Computational Fluid Dynamics, Hermosa Publs., Albuquerque, 1976.

[54] A.A. Samarskii, Local one-dimensional difference schemes on non-uniform nets, J. USSR Comput. Math. Math. Phys. 3 (1963) 572-619.

[55] A.A. Samarskii, B.D. Moiseenko, An economic continuous calculation scheme for the Stefan multidimensional problem, J. Vychisl. Mat. Mat. Fiz. 5 (1965) 816-827.

[56] S.V. Alekseenko, I.S. Anufriev, V.G. Glavniy, D.V. Krasinsky, V.V. Rakhmanov, V.V. Salomatov, E.Y. Shadrin, Study of 3D flow structure and heat transfer in a vortex furnace, Heat Transf. Res. 47 (2016) 653-667. 
[57] V.V. Pomerantsev, Fundamentals of Practical Theory of Combustion, Energoatomizdat, Leningrad, 1986.

[58] A. Fuller, Y. Omidiji, T. Viefhaus, J. Maier, G. Scheffknecht, The impact of an additive on fly ash formation/transformation from wood dust combustion in a lab-scale pulverized fuel reactor, Renew. Energy 136 (2019) 732-745.

[59] J. Ahn, J. Hwan Jang, Combustion characteristics of a 16 step grate-firing wood pellet boiler, Renew. Energy 129 (2018) 678-685.

[60] P. Pradhan, S.M. Mahajani, A. Aror, Production and utilization of fuel pellets from biomass: a review, Fuel Process. Technol. 181 (2018) 215-232.

61] M.E. Mostafa, S. Hu, Yi Wang, S. Su, Xun Hu, S.A. Elsayed, Jun Xiang, The significance of pelletization operating conditions: an analysis of physical and mechanical characteristics as well as energy consumption of biomass pellets, Renew. Sustain. Energy Rev. 105 (2019) 332-348.

[62] A.H. Mahmoudi, F. Hoffmann, M. Markovic, B. Peters, G. t Brem, Numerical modeling of self-heating and self-ignition in a packed-bed of biomass using XDEM, Combust. Flame 163 (2016) 358-369. 